\title{
Mini-commentary on BJOG-20-0640.R1. The evolving definition of pre-eclampsia
}

\author{
Andrew Shennan ${ }^{1}$ and Alice Hurrell ${ }^{2}$ \\ ${ }^{1}$ Kings College London \\ ${ }^{2}$ King's College London
}

November 23, 2020

BJOG-20-0640.R1 The evolving definition of pre-eclampsia

\section{Shennan AH}

\section{Hurrell A}

Pre-eclampsia is a nebulous term. Literally meaning 'prior to a seizure in pregnancy', the name of the syndrome does not accurately depict the clinical reality. The disease rarely leads to eclampsia even if untreated, and this is a rare neurological complication. The definition has been equally imprecise; it has been recognised for many years there is a lack of consistency, even in research papers (Chappell et al.BJOG 1999;106(9)983-5). Original definitions were pragmatic, usually based on consensus, and constructed on blood pressure thresholds known to equate to increased perinatal mortality. The defining link to proteinuria and blood pressure was partly driven by ease of measurement, but clearly there are many other important features of this disease, including renal, hepatic and haematological abnormalities and fetal growth restriction. For this reason, some international organisations have expanded definitions to include evidence of multi-organ and uteroplacental dysfunction (Brown et al. Pregnancy Hypertens.2018;13:291-310). However, there is little validation of these changes, in terms of clinical outcomes and impact.

This paper is welcomed as the authors have an important dataset with well characterised maternal and fetal outcomes, allowing precise methodology for equating definition to outcome (Magee et al. BJOG2020). A traditional definition based on proteinuria thresholds is compared with a broader definition, which includes further maternal symptoms and signs, and is related to important clinical outcomes. Unsurprisingly, this definition produces a higher proportion of women classified as "diseased". This will inevitably improve sensitivity as in effect it lowers the detection threshold. This might seem advantageous as fewer women are "missed", but the trade-off is lower specificity. However, the slight reduction in specificity is likely justified. Individual risk equates to predictive values, and the small change in specificity makes little difference to the positive predictive value ( $46.4 \%$ compared to $44.6 \%)$. The improved sensitivity equates to a substantial benefit in negative predictive value (81.5\% compared to $75.1 \%$ ). Overall, this confirms that the broad definition better distinguishes cases of pre-eclampsia from non-cases and is therefore a better 'rule-out test'. We have calculated predictive values from the authors' primary outcome.

Clinical features remain dependant on end organ response in mother and baby, known to be highly variable in pre-eclampsia. For example, a normal-sized healthy baby is not infrequently found in a severely sick mother and vice versa. Recently, angiogenic biomarkers have been introduced into the NHS. These have better prediction than clinical features and biochemical markers such as urate and liver function when used in women with suspected pre-eclampsia. Definitions may become more accurate in both rule-in and ruleout of adverse events if they incorporate angiogenic markers, as these are likely to reduce false positive and negative assessments of clinical features. If angiogenic biomarkers are used in clinical practice, they 
improve outcomes (Duhig et al. Lancet 2019;393(10183):1807-1818). When a broader definition incorporating angiogenic imbalance and uteroplacental dysfunction is used, this best identifies adverse outcomes (Lai et al.Am J Obstet Gynecol 2020;S0002-9378(20)31286-2). Whether implementation of a broader definition leads to improved outcomes remains to be seen.

Disclosure of interests: AHS has received funds from Perkin Elmer, paid to the university, to evaluate angiogenic markers. AH has nothing to disclose. 